\title{
PFGE and AFLP genotyping of Staphylococcus aureus subsp. anaerobius isolated from goats with Morel's disease
}

\author{
O. Szaluś-Jordanow $\cdot$ D. Chrobak $\cdot$ M. Pyrgiel $\cdot$ A. Lutyńska $\cdot$ J. Kaba $\cdot$ \\ M. Czopowicz • L. Witkowski • M. Kizerwetter-Świda • M. Binek • \\ T. Frymus
}

Received: 10 April 2012/Revised: 20 July 2012/Accepted: 31 August 2012/Published online: 14 September 2012

(C) The Author(s) 2012. This article is published with open access at Springerlink.com

\begin{abstract}
Staphylococcus aureus subsp. anaerobius is the etiological agent of the Morel's disease in sheep and goats. The disease presents with subcutaneous abscesses, located mainly in the superficial lymph nodes. Forty-one isolates of S. aureus subsp. anaerobius were collected from two outbreaks of the Morel's disease in Poland in years 2006-2008. Analysis of DNA SmaI digests by PFGE showed that 35 of 41 isolates belonged to the same PFGE type, identical to the type strain of $S$. aureus subsp. anaerobius ATCC 35844, confirming high level of clonality of the species. The DNA patterns of the remaining identical 6 isolates, different from the reference strain only by two bands, were found closely related. Genotyping performed with AFLP technique revealed two clonal groups including 16 and 25 isolates, respectively. The study indicated that AFLP technique might be a better discriminatory tool for
\end{abstract}

Communicated by Erko Stackebrandt.

O. Szaluś-Jordanow $(\bowtie) \cdot T$. Frymus

Department of Small Animal Disease with Clinic, Faculty

of Veterinary Medicine, Warsaw University of Life Sciences,

Nowoursynowska 159c, 02-776 Warsaw, Poland

e-mail: malaolga@gmail.com; olga_szalus@sggw.pl

D. Chrobak · M. Kizerwetter-Świda · M. Binek

Department of Preclinical Sciences,

Faculty of Veterinary Medicine, Warsaw University

of Life Sciences, Ciszewskiego 8, 02-786 Warszawa, Poland

M. Pyrgiel · A. Lutyńska

Department of Sera and Vaccine Evaluation, National Institute of Hygiene, Chocimska 24, 01-172 Warsaw, Poland

J. Kaba · M. Czopowicz $\cdot$ L. Witkowski

Department of Large Animal Disease with Clinic, Faculty of Veterinary Medicine, Warsaw University of Life Sciences, Nowoursynowska 159c, 02-776 Warsaw, Poland genetic analysis of $S$. aureus subsp. anaerobius isolates, when compared to PFGE.

Keywords PFGE · AFLP .

Staphylococcus aureus subsp. anaerobius .

Goat $\cdot$ Morel's disease

\section{Introduction}

Staphylococcus aureus subsp. anaerobius is the etiological agent of Morel's disease, called also the abscess disease, in sheep and goats. The disease manifests itself with the abscesses located almost exclusively in the superficial lymph nodes or in their close vicinity. The bacterium is closely related to the much more common and medically important pathogen $-S$. aureus subsp. aureus. The main distinguishing feature is complete inability of $S$. aureus subsp. anaerobius to grow under aerobic conditions and lack of catalase activity, which seems to be associated with the pathogenicity of this microorganism (De la Fuente et al. 2010). Methods of genetic analysis developed for S. aureus subsp. aureus may also be applied to the epidemiological investigation of genetic relationships between isolates of $S$. aureus subsp. anaerobius. To date, two different DNA fingerprinting techniques, namely pulsed-field gel electrophoresis (PFGE) and multilocus sequence typing (MLST), have been utilized. They revealed very high homogeneity of isolates coming from Spain, Italy, Denmark, Sudan and Poland (Elhaj and El Sanousi 2005; De la Fuente et al. 2011; Szaluś-Jordanow et al. 2011).

Amplified fragment length polymorphism (AFLP) is a quite novel three-step DNA fingerprinting, developed in early 1990s (Vos et al. 1995). It has been successfully applied to detecting polymorphisms in DNA of many 
different human and animal pathogens (Elderle et al. 1999; Kokotovic et al. 1999; Gzyl et al. 2005), including $S$. aureus subsp. aureus (Cuteri et al. 2004; Gonano et al. 2009). Moreover, it proved to yield results comparable with PFGE and MLST techniques (Melles et al. 2007). Nevertheless, S. aureus subsp. anaerobius has never been analyzed with this method.

Therefore, the study was carried out to compare discriminatory potential of PFGE and AFLP techniques, when applied to the analysis of genetic profile of $S$. aureus subsp. anaerobius.

\section{Materials and methods}

Pathogen isolation

All 41 S. aureus subsp. anaerobius strains were obtained in two goat herds in which outbreaks of Morel's disease were observed in 2006. Both herds had been monitored for 17 months between 2006 and 2008 (Szaluś-Jordanow et al. 2010). At that time, the first herd was visited 9 times and the second herd 7 times, and swabs were collected from all 44 goats in which mature abscesses were detected with clinical examination. Twenty-eight swabs were collected in the first herd, and 26 of them turned out to harbor S. aureus subsp. anaerobius. In the second herd, 16 swabs were collected and $S$. aureus subsp. anaerobius was isolated in 15 cases. The remaining three isolates appeared to be Corynebacterium pseudotuberculosis.

The type strain ATCC 35844 of S. aureus subsp. anaerobius was obtained from LGC Standards. The swabs were cultured on Columbia agar with $5 \%$ sheep blood. Incubation was conducted for $48 \mathrm{~h}$ at $37{ }^{\circ} \mathrm{C}$ under aerobic and microaerophilic conditions. After isolation of pure cultures, identification of $S$. aureus subsp. anaerobius was initially performed basing on the growth only in microaerophilic conditions, microscopic examination (presence of Gram-positive cocci) and biochemical properties (positive coagulase test, negative catalase and clumping factor tests). Biochemical identification was conducted using API-Staph ID (Biomerieux).

\section{PFGE}

Twenty strains, obtained during the initial phase of the study, have already been analyzed using PFGE, and the results were published elsewhere (Szaluś-Jordanow et al. 2011). The remaining 21 strains were cultured on solid ground with blood and then suspended in saline to obtain the density of 3.5 on McFarland scale, centrifuged and mixed with $150 \mu \mathrm{l}$ of PIV buffer and $150 \mu \mathrm{l}$ of liquid agarose. After the solidification, the plugs were placed in
EC buffer and incubated at $37{ }^{\circ} \mathrm{C}$ for $4 \mathrm{~h}$. Then the plugs were incubated in ESP solution at $50{ }^{\circ} \mathrm{C}$ overnight, washed in TE buffer and stored in $1 \mathrm{ml} \mathrm{TE}$ buffer at $4{ }^{\circ} \mathrm{C}$. Next, each plug was placed in $100 \mu \mathrm{l}$ restriction buffer for $15 \mathrm{~min}$. Then each plug was transferred into $250 \mu \mathrm{l}$ of buffer containing $10 \mathrm{U}$ of SmaI and incubated for $3.5 \mathrm{~h}$ at $37{ }^{\circ} \mathrm{C}$ without shaking. The running parameters of the electrophoresis performed with the CHEF-DR II system electrophoresis cell (Biorad) were as follows: initial pulse $-5 \mathrm{~s}$, final pulse- $40 \mathrm{~s}$, voltage $-6 \mathrm{~V} / \mathrm{cm}$, time$20 \mathrm{~h}$ and temperature $-14^{\circ} \mathrm{C}$. The gels were stained with ethidium bromide for $30 \mathrm{~min}$, then washed in distilled water for $30 \mathrm{~min}$, photographed under UV light and documented in the system VersaDoc (Biorad) (Elhaj and El Sanousi 2005). Dendrogram showing relationship strains under study was drawn by cluster analysis with similarity matrices calculated from the Dice product-moment correlation coefficient and UPGMA algorithm (Everitt 1993).

\section{AFLP}

Preliminary screening of different AFLP protocols based on different enzyme restriction/specific adapter ligation and primer-specific amplification with/without selective bases complementary to nucleotides flanking the restriction sites (Vos et al. 1995; Kokotovic et al. 1999; van Elderle et al. 1999; Gzyl et al. 2005) revealed that most informative AFLP patterns for S. aureus subsp. anaerobius strains were obtained with HindIII/TaqI comparing to ApaI/TaqI, HindIII, MfeI/BglII, PstI/TaqI, EcoRI/MseI and SpeI/EcoRI sets tested. Briefly, in the protocol applied to all strains under study, $200 \mathrm{ng}$ of S. aureus subsp. anaerobius DNA was digested with the HindIII (BioLabs Inc.)/TaqI (Gibco BRL), heat-treated and ligated with adapters T4DNA ligase (BioLabs Inc.). Restriction fragments tagged with specific adapters were then selectively amplified with primers (van Elderle et al. 1999). Each amplification reaction mixture contained $5 \mu$ of 10 times diluted sample, $0,5 \mu \mathrm{M}$ of Cy5-labeled primer, 1,7 $\mu \mathrm{M}$ of non-labeled primer, $120 \mu \mathrm{M}$ of dATP, dGTP, dCTP, dTTP each, $0.3 \mathrm{U}$ of AccuTaq-LA DNA polymerase, $3.0 \mathrm{mM}$ of $\mathrm{MgCl}_{2}, 50 \mathrm{mM}$ of TrisHCl, $15 \mathrm{mM}$ of ammonium sulfate and $0.1 \%$ Tween 20. Amplification was performed in the Biometra thermal cycler in conditions published previously (van Elderle et al. 1999). After completion of the PCR, $5 \mu$ of each reaction tube mixture was mixed with $3 \mu \mathrm{l}$ of loading dye denatured at $90{ }^{\circ} \mathrm{C}$ for 3 min and separated through a ReproGel High Resolution (GE Healthcare Bio-Sciences AB, Uppsala) in $0.5 \times \mathrm{TBE}$ buffer on an ALFexpress DNA sequencer (AmershamPharmacia Biotech). Separation was done at $1,500 \mathrm{~V}$, $60 \mathrm{~mA}, 25 \mathrm{~W}$ for $420 \mathrm{~min}$ at $55^{\circ} \mathrm{C}$. To evaluate intra- and inter-gel differences and identity levels, a fluorescein-labeled 
molecular size marker and an external reference strain were used as external size markers. Stored fluorograms were analyzed with the GelCompar Software Version 3.1 (Applied Maths, Kortrijk, Belgium). Dendrogram for cluster analysis was based on similarity matrices calculated from the Pearson product-moment correlation coefficient and UPGMA algorithm (Everitt 1993).

\section{Results}

In PFGE 35 of 41 tested isolates proved to be a single clone A, identical to the type strain of $S$. aureus subsp. anaerobius ATCC 35844. The remaining 6 isolates (112, 611, 272, 941-5, 101 and 130), designated B, differed in the profile from the reference strain only by two bands and were found closely related (Fig. 1). Genetic similarity for strains under study, which represented two different PFGE profiles as evaluated by cluster analysis, was found $68 \%$.

Twenty-three AFLP profiles were identified at the genetic similarity level of $90 \%$, as overall intra- and intergel similarity levels for the external reference strain were

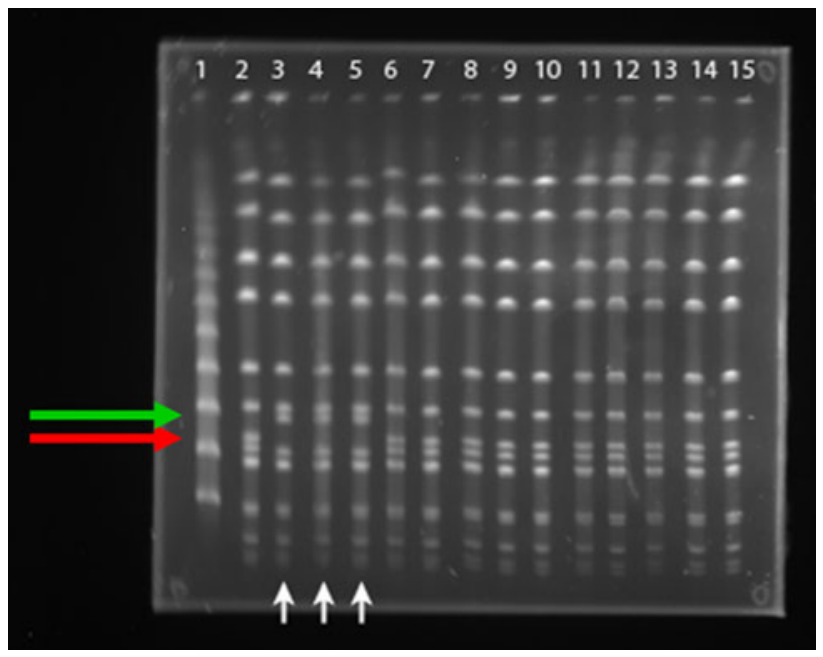

Fig. 1 Macrorestriction analysis of chromosomal DNA of $S$. aureus subsp. anaerobius isolates by PFGE, line 1 molecular weight marker, lines 2-14 isolates of $S$. aureus subsp. anaerobius, line 15 reference strain of S. aureus subsp. anaerobius DSM 20714 (vertical arrows indicate the isolates different from the others by two bands, the upper horizontal arrow indicates the place of the additional band, and the bottom horizontal arrow indicates missing band)
Fig. 2 UPGMA dendrogram constructed from AFLP fingerprints showing genetic relationships between $S$. aureus subsp. anaerobius isolates. Red numbers indicate six different strains in PFGE method (color figure online)

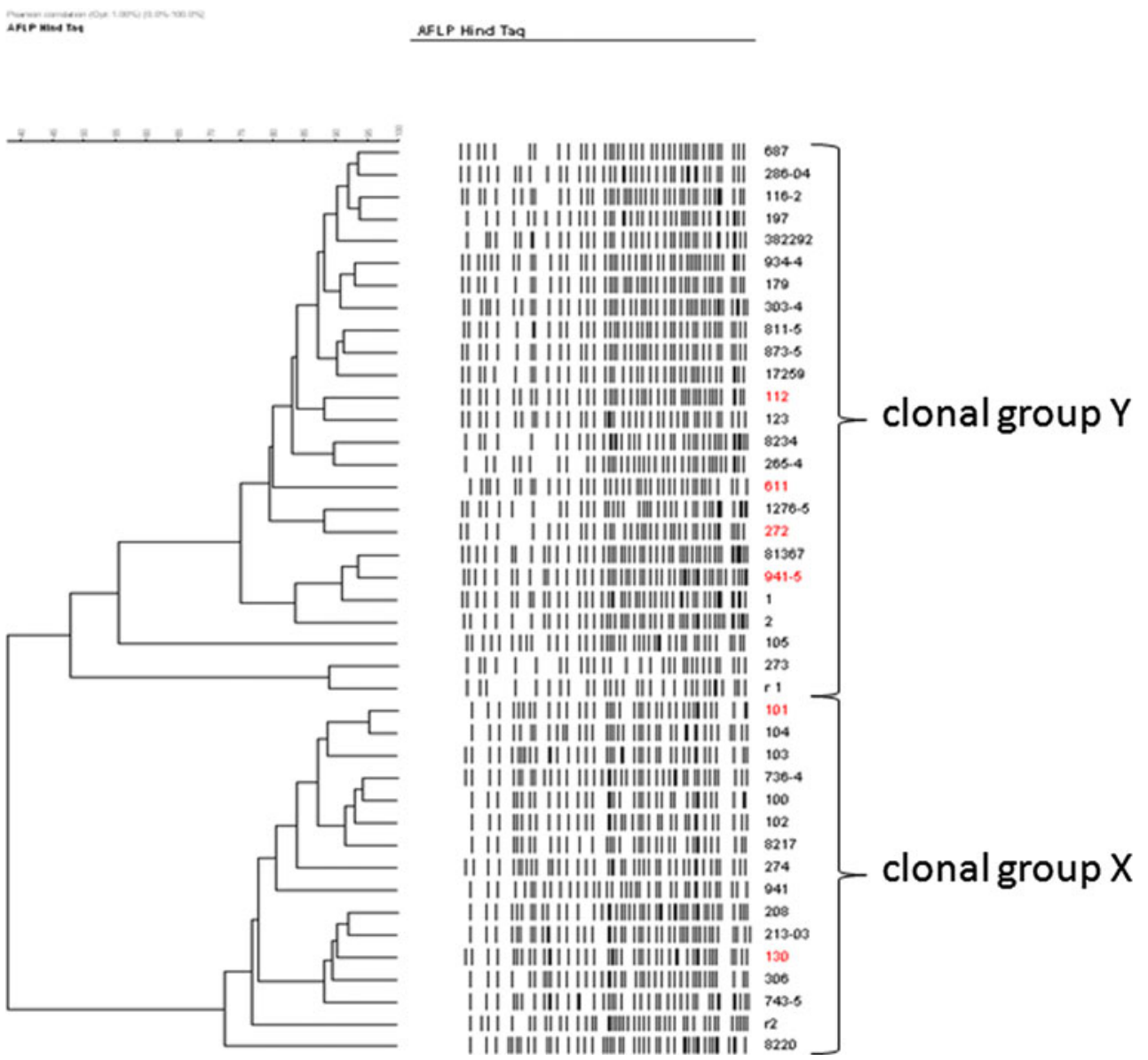


97 and $90 \%$, respectively. Two clonal AFLP groups, X and $\mathrm{Y}$, could be distinguished, containing 16 and 25 isolates, respectively (Fig. 2). Among $\mathrm{X}$ and $\mathrm{Y}$ clonal groups identified, 9 and 14 different AFLP patterns were identified, respectively. Genetic similarity of AFLP patterns of isolates belonging to the clonal group $\mathrm{X}$ was higher $(72 \%)$ than of those belonging to the clonal group Y $(47 \%)$. The overall genetic similarity of the isolates was $38 \%$. In the AFLP clonal group $\mathrm{Y}$ and $\mathrm{X}, 4$ and 2 strains belonging to PFGE group B were found, respectively.

\section{Discussion}

The relationship between isolates of $S$. aureus subsp. anaerobius was analyzed by PFGE using SmaI endonuclease. Previously, PFGE was found to be useful for differentiation of both S. aureus subsp. aureus (Annemüller et al. 1999) and $S$. aureus subsp. anaerobius isolates (Elhaj and El Sanousi 2005; De la Fuente et al. 2011). According to the classification of relationship among isolates by Tenover et al. (1995), 35 of 41 isolates tested in our study were a single clone, identical to the type strain of $S$. aureus subsp. anaerobius ATCC 35844.

The remaining six isolates, which differed from the reference strain only by two bands (Fig. 1), were found closely related. Tiny differences detected by PFGE might result from point mutations, for example, substitution, insertion or deletion of single nucleotides, which could possibly occur in the period of time when disease was present in the herd. Our study confirmed high level of clonality of the species reported previously by Elhaj and El Sanousi (2005) and very recently by De la Fuente et al. (2011). While the former study was conducted on only 6 isolates out of which 4 belonged to the same clone, the latter study included as many as 94 isolates, obtained from four different countries, and hence, its results seem to be very reliable.

Higher level of genetic differentiation of analyzed $S$. aureus subsp. anaerobius strains in comparison with PFGE was obtained using AFLP technique, as overall genetic similarity of the isolates was $38 \%$. Two AFLP clonal groups of different genetic similarity levels identified might suggest that two different clones are actually successfully disseminating among goat herds in Poland. Both AFLP and PFGE are considered to yield comparable results in genetic typing of S. aureus (Melles et al. 2007); nevertheless, no clear distinction could be made with PFGE. Given that the analysis allowed us to discriminate different DNA profiles at the $90 \%$ identity level, AFLP technique seems to be of higher resolution than PFGE in determining genetic profile of $S$. aureus subsp. anaerobius. As in PFGE fingerprinting, strains were differing only by two band positions; both profiles defined among collection under study should be rather considered as subclones. This might be supported by independent position of strains belonging to B PFGE profile within two clusters on the AFLP dendrogram.

Concluding, AFLP technique was found more discriminatory when compared to PFGE fingerprinting. Hence, it may be a useful add-on tool in epidemiological investigation of Morel's disease.

Acknowledgments This work was supported by a grant from the State Committee for Scientific Research, number N N308 025734.

Open Access This article is distributed under the terms of the Creative Commons Attribution License which permits any use, distribution, and reproduction in any medium, provided the original author(s) and the source are credited.

\section{References}

Annemüller C, Lämmler C, Zschöck M (1999) Genotyping of Staphylococcus aureus isolated from bovine mastitis. Vet Microbiol 69:217-224

Cuteri V, Marenzoni ML, Mazzolla R, Tosti N, Merletti L, Arcioni S, Valente C (2004) Staphylococcus aureus: study of genomic similarity of strains isolated in veterinary pathology using amplified fragment length polymorphism (AFLP). Comp Immunol Microbiol Infect Dis 27:247-253

De la Fuente R, Diez RM, Dominguez-Bernal G, Orden JA, MartinezPulgarin S (2010) Restoring catalase activity in Staphylococcus aureus subsp. anaerobius leads to loss of pathogenicity for lambs. Vet Res 41:41

De la Fuente R, Ballesteros C, Bautista V, Medina A, Orden JA, Domínguez-Bernal G, Vindel A (2011) Staphylococcus aureus subsp. anaerobius isolates from different countries are clonal in nature. Vet Microbiol 150:198-202

Elderle J, Janssen P, Hoefnagels-Schuermans A, van Lierde S, Peetermans WE (1999) Amplified-fragment length polymorphism analysis versus macro-restriction fragment analysis for molecular typing of Streptococcus pneumoniae isolates. J Clin Microbiol 37:2053-2057

Elhaj M, El Sanousi SM (2005) Pulsed-field gel electrophoresis for comparison of Staphylococcus aureus subsp. anaerobius local Sudanese isolates. J Anim Vet Adv 8:706-707

Everitt B (1993) Cluster analysis, 3rd edn. Arnold, London

Gonano M, Hein I, Zangerl P, Rammelmayr A, Wagner M (2009) Phenotypic and molecular characterization of Staphylococcus aureus strains of veterinary, dairy and human origin. Epidemiol Infect 137:688-699

Gzyl A, Augustynowicz E, Mosiej E, Zawadka M, Gniadek G, Nowaczek A, Slusarczyk J (2005) Amplified fragment length polymorphism (AFLP) versus random amplified polymorphic DNA (RAPD) as a new tools for inter- and intra-species differentiation within Bordetella. J Med Microbiol 54:333-346

Kokotovic B, Friis F, Jensen JS, Ahrens P (1999) Amplified-fragment length polymorphism fingerprinting of Mycoplasma species. J Clin Microbiol 37:3300-3307

Melles DC, van Leeuwen WB, Snijders SV, Horst-Kreft D, Peeters JK, Verbrugh HA, van Belkum A (2007) Comparison of multilocus sequence typing (MLST), pulsed-field gel electrophoresis (PFGE), and amplified fragment length polymorphism 
(AFLP) for genetic typing of Staphylococcus aureus. J Microbiol Methods 69:371-375

Szaluś-Jordanow O, Kaba J, Czopowicz M, Witkowski L, Nowicki M, Nowicka D, Stefańska I, Rzewuska M, Sobczak-Filipiak M, Binek M, Frymus T (2010) Epidemiological features of Morel's disease in goats. Pol J Vet Sci 13:437-445

Szaluś-Jordanow O, Kanbar T, Soedarmanto I, Eissa N, Alber J, Lämmler C, Zschöck M, Weiss R, Kaba J, Frymus T (2011) Phenotypic and genotypic properties of Staphylococcus aureus subsp. anaerobius isolated from lymph node abscesses of goats. Berl Munch Tierarztl Wochenschr 124:123-127
Tenover FC, Arbeit RD, Goering RV, Mick PA (1995) Interpreting chromosomal DNA restriction patterns produced by pulsed-field gel electrophoresis: criteria for bacterial strain typing. J Clin Microbiol 33:2233-2239

Vos P, Hogers R, Bleeker M, Reijans M, van de Lee T, Hornes M, Frijters A, Pot J, Peleman J, Kuiper M, Zabeau M (1995) AFLP: a new technique for DNA fingerprinting. Nucleic Acids Res 23:4407-4414 